\title{
Laser de femtosegundo versus microceratótomo mecânico na confecção do flap para LASIK
}

\author{
Femtosecond laser versus mechanical microkeratome for LASIK flap creation
}

\author{
Alexander Rodrigo Hasimoto ${ }^{1}$, Manuela Fiorese Benites Gomes ${ }^{1}$, Marcelo Alves Vilar de Siqueira², Hamilton Moreira ${ }^{3}$
}

\section{RESUMO}

Objetivo: Avaliar comparativamente os resultados cirúrgicos, na técnica LASIK, realizando com laser de femtosegundo e microcerátomo mecânico.

Métodos: Estudo clínico, prospectivo, comparativo, randomizado e mascarado de 32 olhos (16 pacientes). Cada paciente foi submetido a diferentes técnicas para - LASIK, em um dos olhos foi utilizado o microcerátomo Hansatome ${ }^{T M}$ Bausch \& Lomb (grupo microcerátomo) e no outro foi utilizado femtosegundo FEMTO LDV TM Ziemer (grupo femtosegundo). A triagem ocorreu no setor de Cirurgia Refrativa do Hospital de Olhos do Paraná entre 07/2010 e 09/2010. Os critérios de inclusão foram miopia menor que 6,00 D, astigmatismo menor que 3,00 D, menor que 5,00 D de hipermetropia, refração estável, diâmetro corneano menor que $11 \mathrm{~mm}$, descontinuação de lente de contato sete dias antes da avaliação pré-operatória acuidade visual de no mínimo 20/20 em ambos os olhos. As variáveis analisadas no pré-operatório entre os grupos, femtosegundo e microcerátomo foram: acuidade visual sem correção e com correção, equivalente esférico, aberrações totais de alta ordem, acuidade visual de baixo contraste, preferência do paciente e complicações. Resultados: Todas as variáveis estudadas foram similares nos dois grupos.

Conclusão: Não foi possível observar neste estudo, diferenças significativas entre os grupos analisados

Descritores: Ceratomileuse assistida por excimer laser in situ/métodos; Ceratectomia fotorrefrativa/métodos; Lasers de excimer;Miopia/cirurgia; Acuidade visual/ fisiologia

\begin{abstract}
Purpose: To assess LASIK flaps made by femtosecond laser and mechanical microkeratome.

Methods: Clinical, prospective, randomized, masked study of 32 eyes (16 patients). Both eyes of all patients were operated, each patient underwent different techniques for lasik. Microkeratome Hansatome $e^{T M}$ Bausch \& Lomb (group microkeratome) was used in one eye and femtosecond laser Femto LDV VM Ziemer (group femtosecond) was used for the fellow eye. Patients were selected from the Refractive Surgery service of the Eye Hospital of Paranábetween July 2010 and September 2010. Inclusion criteria were myopia less than $6.00 \mathrm{D}$, astigmatism less than $3.00 \mathrm{D}$, hyperopia less than $5.00 \mathrm{D}$, stable refraction over one year, corneal diameter smaller than $11 \mathrm{~mm}$, discontinuation of contact lenses seven days before the preoperative evaluation, corrected visual acuity of at least 20/20. Eyes were randomly allocated for each technique. The studied variables were: visual acuity with and without correction, residual refractive error, high order aberrations, low contrast visual acuity, complications and subjective patient preference.
\end{abstract}

Results: All studied variables were similar between the two groups.

Conclusion: We could not demonstrate any difference between the studied groups.

Keywords: Keratomileusis, laser in situ/methods; Photorefractive keratectomy/methods; Lasers, excimer; Myopia/surgery; Visual acuity/physiology

\section{INTRODUÇÃO}

O LASIK (Laser in situ keratomileusis) é uma das cirurgias refrativas corneanas mais comuns usadas para correção de miopia. Recentemente, uma nova tecnologia está revolucionando os mais variados tipos de cirurgias corneanas. O laser de femtosegundo foi introduzido para criação de "flaps" precisos ${ }^{(1,2)}$, um passo crítico no procedimento cirúrgico. Além de determinar um novo patamar de segurança e qualidade no LASIK, o femtosegundo tem sido usado para várias intervenções corneanas, destacando-se o transplante de córnea e a criação de túneis para implante dos segmentos de anéis intracorneanos no tratamento do ceratocone. Ele utiliza um pulso ultrarrápido, menos de um quadrilhão de segundo $\left(10^{15}\right.$ de segundo), de luz infravermelha, que induz a uma fotodisrupção, ou seja, a divisão de um material a nível molecular sem que ocorra grande transferência de calor ou impacto ${ }^{(1,3)}$. Tanna et al., descrevem os resultados precoces no "flap" criado por femtosecond X microcerátomo no LASIK(3). No entanto, muito poucos estudos mostram o resultado à longo prazo comparando os dois métodos na criação do "flap".

\section{MÉTODOS}

Este foi um estudo clínico, prospectivo, comparativo, randomizado e mascarado (com relação ao paciente, este não sabia qual técnica cirúrgica seria impregada em cada olho e em relação ao avaliador, não detinha conhecimento prévio da técnica impregada em cada olho) de 32 olhos (16 pacientes). Foram operados ambos os olhos de todos os pacientes, cada paciente foi submetido a diferentes técnicas para o LASIK, em um dos olhos foi utilizado o microcerátomo Hansatome ${ }^{T M}$ Bausch \& Lomb (grupo microcerátomo) e no outro olho foi utilizado femtosegundo FEMTO LDV TM Ziemer (grupo femtosegundo). A triagem e seleção dos pacientes ocorreu no setor de Cirurgia Refrativa do Hospital de Olhos do Paraná (HOPR) entre julho de 2010 e setembro de 2010. Esta pesquisa foi aprovada pelo comitê de ética em pesquisa do HUEC sendo obtido consentimento livre e esclarecido de todos os participantes.

Os critérios de inclusão foram miopia menor que 6,00 D, astigmatismo menor que 3,00, e hipermetropia menor que 5,00 D, refração estável, diâmetro corneano menor que $11 \mathrm{~mm}$, descontinuação de
Financiamento: Não houve financiamento para este trabalho.

Divulgação de potenciais conflitos de interesse: A.R.Hasimoto, Nenhum; M.F.B.Gomes, Nenhum; M.A.V.Siqueira, Nenhum; H.Moreira, Nenhum.

Endereço para correspondência: Alexander Rodrigo Hasimoto. Rua Benjamin Constant, 788 - Ponta Grossa (PR) - 84010-380 - Brasil - E-mail: tosa_alex@hotmail.com

Protocolo número 2814 - Comitê de Ética em Pesquisa da Sociedade Evangélica Beneficiente de Curitiba. 
lente de contato sete dias antes da avaliação pré-operatória, acuidade visual corrigida de 20/20 em ambos os olhos.

Os critérios de exclusão foram o uso de lente rígida gás permeável, olho seco e blefarite severos, anormalidades do segmento anterior (catarata, cicatriz corneana, neovascularização dentro de $1 \mathrm{~mm}$ da área de ablação), erosão corneana recorrente, doença da membrana basal severa, ceratocone ou miopia progressiva ou instável, miras corneanas instáveis na ceratometria central e espessura corneal em que resulte menos de 250 micra no leito residual, avaliação da topografia e/ou aberrações pré-operatórias que indiquem olhos nãoaptos para cirurgia de correção visual de LASIK (ceratocone forma frusta, "warpage" corneano, degeneração marginal pelúcida), cirurgia intraocular ou corneana prévia, história de ceratite por herpes, uso de corticoide sistêmico em dose imunosupressiva, pacientes imunocomprometidos com doença atópica significativa, doença do tecido conectivo, diabetes melitus, anormalidades maculares, gravidez ou lactação, participação em outros testes $\operatorname{clínicos}^{(1,4)}$.

A escolha do olho e da técnica cirúrgica para confecção seguiu ordem aleatória (através de envelopes opacos enumerados por ordem de chegada dos pacientes, contendo dentro dois papéis com o nome da técnica a ser utilizada e outro envelope opaco contendo dois papéis com olho direito e olho esquerdo para ambos serem sorteados; a partir do momento em que foi definida uma técnica cirúrgica para determinado olho, o olho contralateral recebeu a outra técnica). No pré-operatório os pacientes foram submetidos aos exames de acuidade visual sem correção, acuidade visual com correção, biomicroscopia, mapeamento de retina, tonometria, paquimetria, teste de sensibilidade ao contraste (teste Pelli-Robson, iniciando pelo olho direito com correção no pré-operatório e sem correção no pósoperatório e com dilatação, tamanho de letra (optotipo 20/60), distância de teste de 2 metros e a iluminação ambiente de 85 candelas $/ \mathrm{m}^{2}$ ), aberrometria (LadarWave ${ }^{\circledR}$ Alcon, Fort Worth, Texas), topografia corneana (EyeSys Vision, Inc. V 4.5)(1).

O tratamento foi feito com excimer laser (WaveLight ${ }^{\circledR}$ Allegretto Wave ${ }^{\circledR}$ Eye-Q Laser, Alcon Surgical, Fortworth, EUA), por um único cirurgião. A lamela do laser de femtosegundo foi programada para $110 \mu \mathrm{m}$ de espessura e 9,5 mm de diâmetro, a do microcerátomo mecânico foi programada para $160 \mu \mathrm{m}$ de espessura e 9,5 mm de diâmetro. As cirurgias dos dois olhos foram feitas sequencialmente no mesmo ato cirúrgico e se iniciaram conforme a escolha aleatória dos envelopes. No pós-operatório todos os pacientes foram examinados, por um único médico examinador, que não soube qual técnica foi utilizada na cirurgia. Os pacientes receberam atendimento no $1^{\circ} \mathrm{dia}$, 1a semana, 1 e 3 meses após LASIK ${ }^{(1)}$. Todos os pacientes foram submetidos à avaliação de acuidade visual e exame de lâmpada de fenda. No 3 mês pós-operatório, foram realizados os exames de acuidade visual sem correção, refração, teste de sensibilidade ao contraste, topografia corneana, aberrometria, biomicroscopia, paquimetria.

Para análise estatística foi utilizado o teste não-paramétrico "Wilcoxon" (através do software R Core Team (2013), versão 3.0.1). O nível de significância adotado foi menor que 5\% $(p<0,05)$.

\section{RESULTADOS}

Os pacientes foram submetidos a técnicas cirúrgicas para confecção do "flap", sendo 16 olhos por microcerátomo mecânico Hansatome (Grupo 1) e 16 olhos por laser de femtosegundo (Grupo 2). Na comparação entre os grupos, em relação a idade, não foi observado diferença significativa.

O tempo de evolução (período compreendido entre o pré e o pós-operatório) foi de 157,5 \pm 36,7 dias, variando de 117,0 a 255,0 dias, sendo o mesmo para os grupos avaliados. O acompanhamento pós-operatório foi feito no 1, 7, 30 e 90 dias e em todos eles os 16 pacientes foram examinados. De um total de 19 pacientes propostos para o estudo, 3 pacientes (6 olhos) foram descontinuados, pois apresentaram complicações. Um paciente sofreu trauma contuso em um olho no 10ำ pós-operatório. Imediatamente o paciente sofreu intervenção cirúrgica para reposicionamento do "flap". Ao final do terceiro mês apresentava acuidade visual sem correção de 20/16 neste olho. Um paciente apresentou ceratite lamelar difusa (DLK), em apenas um olho (grupo femtosegundo), ao final do terceiro dia. Foi necessária intervenção cirúrgica, apresentando boa evolução, com acuidade visual não corrigida de 20/25 neste olho. E o último paciente apresentou células hemáticas na interface do "flap", sendo necessária a lavagem deste, com boa evolução e acuidade visual sem correção de 20/20 no final do estudo. Assim, todo estudo baseou-se na avaliação de 16 pacientes.

Em relação a acuidade visual, todos os pacientes possuíam no pré-operatório 20/20 ou melhor. Comparando a acuidade visual não-corrigida, no primeiro dia $(p=0,63)$, sétimo $(p=0,72)$, trigésimo dia $(p=0,83)$ e três meses $(p=0,89)$, os resultados se equivaleram, diferença não-significativa (teste de Wilcoxon) (Figura 1).

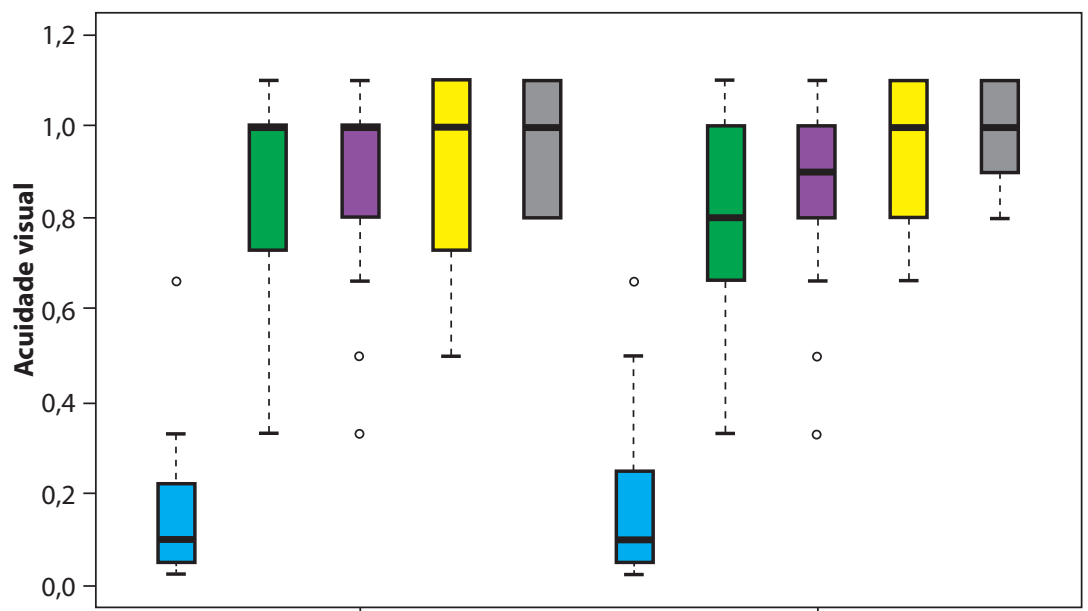

Femtosegundo

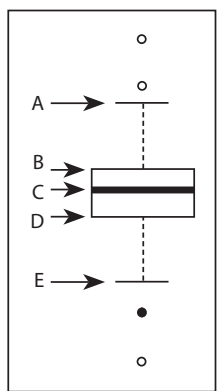

A: é a maior observação do conjunto de dados que é menor que $B+(B-D) \times 1,5$

$B:$ é o terceiro quartil do conjunto de dados. C: é a mediana do conjunto de dados.

D: é o primeiro quartil do conjunto de dados.

E: é a menor observação do conjunto de dados que é maior que $D$ - $(B-D) \times 1,5$
$\square$ Pré-operatório
$\square$ Pós-operatório de 1 dia
$\square$ Pós-operatório de 7 dias
$\square$ Pós-operatório de 30 dias
$\square$ Pós-operatório de 90 dias

Figura 1. Acuidade visual sem correção no pré e pós-operatório. 
O equivalente esférico médio no pós-operatório de três meses no grupo femtosegundo foi de $-0,75$ e no grupo microcerátomo de -0,50. Em 97\% dos olhos operados, apresentavam-se dentro de $1 \mathrm{D}$ da mudança refracional programada (Figura 2).

As aberrações totais de alta-ordem, ao terceiro mês, foram similares em ambos os grupos, não havendo diferença significativa. ( $p=0,84$ - teste de Wilcoxon) (Figura 3), medida obtida em uma pupila de $6,5 \mathrm{~mm}$.

Em relação a acuidade visual de baixo contraste, foi observado na análise comparativa entre os grupos, em relação à frequência espacial pré-operatória, que não houve diferença significativa (Tabela 1). Na avaliação pós-operatória entre os grupos femtosegundo e microcerátomo, houve diferença significativa somente na frequência espacial de 1,5 ciclos por grau ( $p=0,04$ - teste de Wilcoxon) (Tabela 2).

Em relação ao conforto e preferência do paciente, apesar de grande parte dos pacientes referir mais conforto no olho do microcerátomo durante o procedimento e maior preferência no olho do femtosegundo no primeiro e sétimo dia, os resultados foram estatisticamente não significantes.

\section{DISCUSSÃO}

O laser de femtosegundo foi desenvolvido para utilização em vários procedimentos na oftalmologia. Está sendo utilizado para confecção de transplantes lamelares, túneis para implante de anéis intraestromais, "pockets" para "corneal inlays" e em alguns passos da

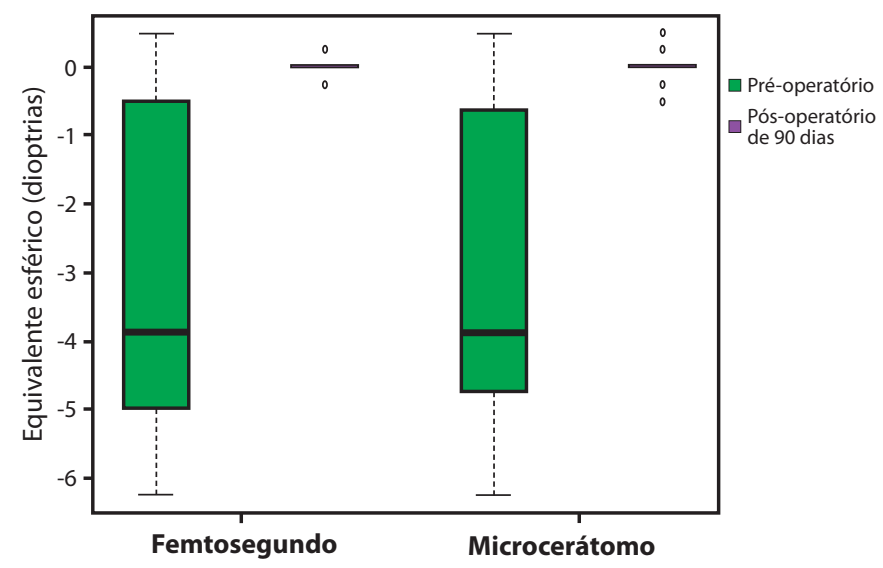

Figura 2. Equivalente esférico no pré e pós-operatório.

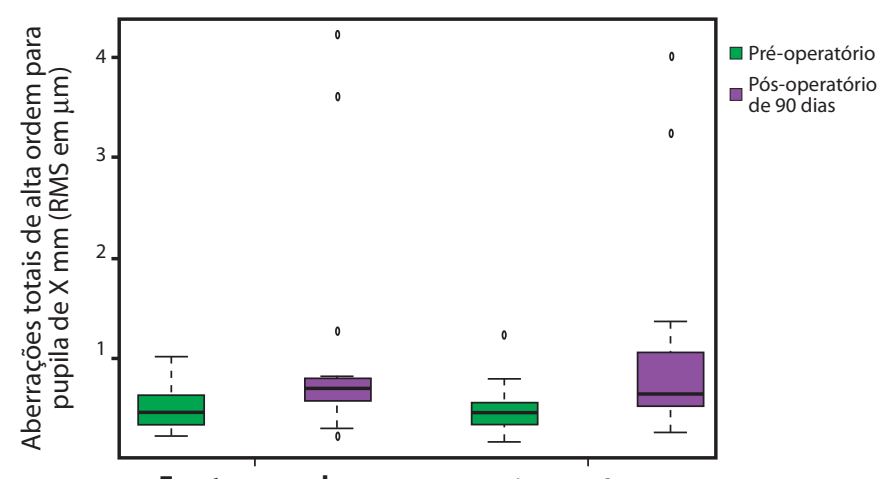

Femtosegundo

Microcerátomo

Figura 3. Aberrações totais de alta-ordem no pré e pós-operatório. facoemulsificação. Para a grande parte destes procedimentos a superioridade do femtosegundo quando comparado à técnica convencional é nítida. Para o LASIK, porém, apesar de existirem estudos comparando o laser de femtosegundo com o microcerátomo mecânico, ainda há divergências sobre a preferência tanto do cirurgião quanto do paciente em relação ao procedimento escolhido ${ }^{(3,5-7)}$.

O presente estudo analisou de forma prospectiva os resultados de "flaps" confeccionados pelo laser de femtosegundo em um olho e por microcerátomo mecânico no olho contralateral.

Quando observado o fator conforto durante o procedimento cirúrgico, houve uma preferência para o olho em que foi utilizado o microcerátomo mecânico. Em nosso estudo, alguns pacientes relataram maior desconforto no acoplamento e no momento da utilização do vácuo nos olhos que foram submetidos ao laser de femtosegundo. Porém este resultado não foi significante estatisticamente. Em relação a fotofobia, apesar de esta ser uma sensação transitória relatada em 1 a 2 \% dos casos de criação do "flap" com femtosegundo a incidência cai para menos de $0,25 \%$ quando é utilizada $20 \%$ menos energia. A causa parece ser decorrente de uma resposta inflamatória tardia. Em 30\% dos pacientes que desenvolvem ceratite lamelar difusa, a sensibilidade à luz transitória também vai acontecer. Esteroides tópicos são um tratamento adequado para as duas situações ${ }^{(8)}$. Outro estudo também questionou a preferência dos pacientes após os três meses de seguimento e observou que de 21 pacientes (42 olhos), 5 preferiram o olho em que não foi utilizada lâmina, 7 o do olho do microcerátomo e 9 não preferiram nenhuma das técnicas ${ }^{(9)}$.

Não houve diferença significativa na medida da acuidade visual entre os grupos. Em 20.000 casos observados em 2009, comparando as duas técnicas cirúrgicas em questão, foi confirmado que a recuperação visual no "flap" com femtosegundo é mais rápida, mas o resultado nos dois grupos é similar após seis meses ${ }^{(10)}$.

Tabela 1. Análise comparativa do período pré-operatório entre os grupos 1 (femtosegundo) e 2 (microcerátomo)

\begin{tabular}{|c|c|c|c|}
\hline \multirow{2}{*}{$\begin{array}{l}\text { Teste de sensibilidade } \\
\text { ao contraste (log) }\end{array}$} & $\begin{array}{c}\text { Grupo } 1 \\
\text { (femtosegundo) } \\
\end{array}$ & $\begin{array}{c}\text { Grupo } 2 \\
\text { (microcerátomo) } \\
\end{array}$ & \multirow[b]{2}{*}{ Valor-p ${ }^{(1)}$} \\
\hline & $M \pm D V$ & $M \pm D V$ & \\
\hline Frequência espacial 1,5cpg & $0,75 \pm 0,06$ & $0,74 \pm 0,04$ & 0,89 \\
\hline Frequência espacial 3 cpg & $0,78 \pm 0,04$ & $0,79 \pm 0,07$ & 0,90 \\
\hline Frequência espacial 6 cpg & $0,72 \pm 0,07$ & $0,72 \pm 0,08$ & 0,78 \\
\hline Frequência espacial 12 cpg & $0,61 \pm 0,15$ & $0,63 \pm 0,13$ & 0,35 \\
\hline Frequência espacial 18 cpg & $0,58 \pm 0,14$ & $0,58 \pm 0,14$ & 0,80 \\
\hline
\end{tabular}

$\mathrm{M} \pm \mathrm{DV}=$ média \pm desvio padrão; $\mathrm{cpg}=$ ciclos por grau.

(1)= valor-p do teste de Wilcoxon para amostras pareadas (dependentes)

Tabela 2. Análise comparativa do período pós-operatório entre os grupos 1 (femtosegundo) e 2 (microcerátomo)

\begin{tabular}{|c|c|c|c|}
\hline \multirow{2}{*}{$\begin{array}{l}\text { Teste de sensibilidade } \\
\text { ao contraste (log) }\end{array}$} & $\begin{array}{c}\text { Grupo } 1 \\
\text { (femtosegundo) } \\
\end{array}$ & $\begin{array}{c}\text { Grupo } 2 \\
\text { (microcerátomo) } \\
\end{array}$ & \multirow[b]{2}{*}{ Valor-p ${ }^{(1)}$} \\
\hline & $M \pm D V$ & $M \pm D V$ & \\
\hline Frequência espacial 1,5cpg & $0,74 \pm 0,06$ & $0,69 \pm 0,08$ & $0,04^{*}$ \\
\hline Frequência espacial 3 cpg & $0,78 \pm 0,05$ & $0,76 \pm 0,06$ & 0,11 \\
\hline Frequência espacial 6 cpg & $0,70 \pm 0,11$ & $0,69 \pm 0,13$ & 1,00 \\
\hline Frequência espacial 12 cpg & $0,59 \pm 0,22$ & $0,63 \pm 0,13$ & 0,47 \\
\hline Frequência espacial 18 cpg & $0,59 \pm 0,12$ & $0,52 \pm 0,24$ & 0,31 \\
\hline
\end{tabular}

$\mathrm{M} \pm \mathrm{DV}=$ média \pm desvio padrão; $\mathrm{cpg}=$ ciclos por grau.

${ }^{(1)}=$ valor-p do teste de Wilcoxon para amostras pareadas (dependentes).

* = diferença significativa a um nível de 5\%. 
Em relação à sensibilidade ao contraste não houve diferença significativa entre os dois grupos. Em outro estudo, também foi atestado que não houve diferença do pré-operatório ou em qualquer pós-operatório em nenhuma frequência espacial. Na maior frequência espacial (18 ciclos por grau), a sensibilidade ao contraste aumentou nos dois grupos com três meses de pós-operatório de forma desigual (femtosegundo $\mathrm{p}=0,02 \%$ e microcerátomo mecânico $p<0,001$ ), mas não foi detectada diferença após seis meses em nenhum dos tratamentos ${ }^{(9)}$.

Em estudo realizado por Netto et al., foi comparada a resposta cicatricial e inflamatória do leito estromal das duas formas de confecção do flap. O resultado obtido mostrou que a necrose de células estromais associada com a formação do "flap" com o laser de femtosegundo pode contribuir para uma maior inflamação do LASIK realizado desta forma, especialmetne com maiores níveis de energia que resultam em maior morte dos ceratócitos. Desta forma o laser de $60 \mathrm{kHz}$ e o microcerátomo não tiveram diferença significativa em nenhum parâmetro nas primeiras 24 horas, exceto pela mitose das células estromais na margem do "flap"(11). Em 2008 Jones et al., comparou o leito estromal após a ceratoplastia endotelial em olhos doadores realizados com laser de femtosegundo de $30 \mathrm{kHz}$ e microcerátomo mecânico e constatou que os dois são igualmente efetivos na criação do pré corte da ceratoplastia endotelial do olho doador, sem efeito significativo na densidade de células endoteliais. A única diferença que encontrou foi a criação de uma superfície mais irregular no "flap" do femtosegundo, o que poderia aumentar a aderência do disco beneficiando a cirurgia ${ }^{(12)}$.

Finalmente quando analisamos as aberrações de alta ordem antes e depois do LASIK realizado nos dois grupos foi observado que não houve diferença significativa. Estudos mostraram a diferença entre os perfis de aberrações de alta ordem que foram favoráveis aos "flaps" de femtosegundo ${ }^{(13)}$. Medeiros et al. ${ }^{(13)}$, diz que devido ao femtosegundo produzir um "flap" mais plano, ele pode reduzir levemente as aberrações de alta ordem se comparado com o "flap" do microcerátomo mecânico que apresenta forma de menisco. No entanto, sem diferenças significativas. Estas aberrações são mais fortemente associadas com a própria ablação do laser. Na França, analisaram comparativamente estas duas técnicas em relação a previsibilidade da profundidade do "flap", efeitos biomecânicos da córnea e aberrações ópticas. A previsibilidade do "flap" foi maior no grupo do femtosegundo, porém as propriedades biomecânicas da córnea foram menores nos dois grupos quando comparados com o pré-operatório. Já as aberrações de alta ordem aumentaram após o LASIK em relação ao pré-operatório e não foram influenciadas pela técnica de confecção do "flap"(14).

\section{CONCLUSÃO}

Concluímos que apesar do laser de femtosegundo ser um recurso tecnológico muito interessante para a confecção do "flap" do LASIK, não foi observado em nosso estudo diferença significativa nos dados avaliados. Portanto podemos assegurar que o microcerátomo mecânico também nos confia bons resultados. Porém mais estudos com seguimento maior são necessários.

\section{REFERÊNCIAS}

1. Chan A, Ou J, Manche EE. Comparison of the femtosecond laser and mechanical keratome for laser in situ keratomileusis. Arch ophthalmol. 2008;126(11):1484-90.

2. Hamilton DR, Johnson RD, Lee N, Bourla N. Differences in the corneal biomechanical effects of surface ablation compared with laser in situ keratomileusis using a microkeratome or femtosecond laser. J Cataract Refract Surg. 2008;34(12):2049-56.

3. Tanna M, Schallhorn SC, Hettinger KA. Femtosecond laser versus mechanical microkeratome: a retrospective comparison of visual outcomes at 3 months. J Refract Surg. 2009;25(7):S668-71

4. Lim T, Yang S, Kim M, Tchah H. Comparison of the IntraLase femtosecond laser and mechanical microkeratome for laser in situ keratomileusis. Am J Ophthalmol. 2006; 141(5):833-9.

5. Li H, Sun T, Wang M, Zhao J. Safety and effectiveness of thin-flap LASIK using a femtosecond laser and microkeratome in the correction of high myopia in Chinese patients. J Refract Surg. 2010;26(2):99-106.

6. Montés-Micó R, Rodríguez-Galietero A, Alió JL. Femtosecond laser versus mechanical keratome LASIK for myopia. Ophthalmology. 2007;114(1):62-8.

7. Chen S, Feng Y, Stojanovic A, Jankov MR $2^{\text {nd }}$, Wang Q. IntraLase femtosecond laser vs mechanical microkeratomes in LASIK for myopia: a systematic review and metaanalysis. J Refract Surg. 2012;28(1):15-24.

8. Stonecipher K, Dishler JG, Ignacio TS, Binder PS. Transient light sensitivity after femtosecond laser flap creation: clinical findings and management. J Cataract Refract Surg. 2006;32(1):91-4.

9. Patel SV, Maguire LJ, McLaren JW, Hodge DO, Bourne WM. Femtosecond laser versus mechanical microkeratome for LASIK: a randomized controlled study. Ophtalmology. 2007;114(8):1482-90.

10. Wevill M. Plasma VS steel; results of more than 20.000 casos. Paper presented at: $27^{\circ}$ Annual Congress of the European Society of Cataract \& Refractive Surgeons; 2009 Sep 12-16; Barcelona, Spain.

11. Netto MV, Mohan RR, Medeiros FW, Dupps WJ Jr, Sinhá S, Krueger RR, et al. Femtosecond laser and microkeratome corneal flaps: comparison of stromal woud healing and inflammation. J Refract Surg. 2007;23(7):667-76.

12. Jones YJ, Goins KM, Sutphin JE, Muilins R, Skeie JM. Comparison of the femtosecond laser (IntraLase) versus manual microkeratome (Moria ALTK) in dissection of the donor in endothelial keratoplasty: initial study in eye bank eyes. Córnea. 2008;27(1):88-93.

13. Medeiros FW, Stapleton WM, Hammel J, Krueger RR, Netto MV, Wilson SE. Wavefront analysis comparison of LASIK outcomes with the femtosecond laser and mechanical microkeratomes. J Refract Surg. 2007;23(9):880-7.

14. Kouassi FX, Blaizeau M, Buestel C, Schweitzer C, Gallois A, Colin J, et al. [Comparison of Lasik with femtosecond laser versus Lasik with mechanical microkeratome: predictability of flap depth, corneal biomechanical effects and optical aberrations]. J Fr Ophtalmol. 2012;35(1):2-8. French. 